\title{
Substrate-limited electron dynamics in graphene
}

\author{
S. Fratini ${ }^{1,2}$ and F. Guinea ${ }^{2}$ \\ ${ }_{1}^{1}$ Institut Néel-CNRS and Université Joseph Fourier, Boîte Postale 166, F-38042 Grenoble Cedex 9, France \\ ${ }^{2}$ Instituto de Ciencia de Materiales de Madrid, CSIC, Sor Juana Inés de la Cruz 3, E-28049 Madrid, Spain
}

(Received 26 March 2008; published 13 May 2008)

\begin{abstract}
We study the effects of polarizable substrates such as $\mathrm{SiO}_{2}$ and $\mathrm{SiC}$ on the carrier dynamics in graphene. We find that the quasiparticle spectrum acquires a finite broadening due to the long-range interaction with the polar modes at the interface between graphene and the substrate. This mechanism results in a density dependent electrical resistivity, which exhibits a sharp increase around room temperature, where it can become the dominant limiting factor of electron transport. The effects are weaker in doped bilayer graphene due to the more conventional parabolic band dispersion. Amorphous substrates, such as polymethyl methacrylate, can induce a room temperature resistivity of comparable magnitude, although with a weaker temperature dependence.
\end{abstract}

DOI: 10.1103/PhysRevB.77.195415

PACS number(s): 71.20.Tx, 72.10.Di, 73.63.-b

\section{INTRODUCTION}

Remote phonon scattering is a known limiting factor of the electron mobility in two-dimensional artificial structures of technological interest, such as $\mathrm{Si}$ metal-oxidesemiconductor field-effect transistors (MOSFETs). ${ }^{1-4}$ Due to the polar nature of the gate dielectrics used in such devices, the carriers in the conducting channel electrostatically couple to the long-range polarization field created at the conductor and/or dielectric interface. The interaction primarily occurs with optical phonon modes of high frequency (50-200 meV), which do not affect transport at low temperatures. However, it results in a sizable degradation of the mobility at room temperature (i.e., the temperature of device operation) and becomes the dominant scattering mechanism in devices with high- $\kappa$ gate dielectrics.

The interaction with the phonons of the gate dielectric has been recently investigated in another class of electronic devices, where the Si inversion layer is replaced by a crystalline organic semiconductor. ${ }^{5,6}$ In organic field-effect transistors, due to the narrow-band nature of the active material (typical bandwidths are less than $0.5 \mathrm{eV}$ ), the energy scale associated with the remote phonon interaction can become comparable with the bandwidth, which results in carrier selflocalization. In this case, the effect is more dramatic than in Si MOSFETs: the mobility is not only strongly reduced by the interactions, but it also acquires an exponentially activated temperature dependence due to the hopping motion of the localized carriers.

Graphene-an atomically thin sheet of carbon atoms-is another system where the long-range coupling to the polar modes of the substrate can have a sizable influence on carrier dynamics. Compared to the two-dimensional electron gas formed in Si inversion layers, the effect here is enhanced due to the poor screening properties of the quasiparticles close to the nodal points, which behave as the Dirac fermions. Although most experimental studies of transport in graphene up until now have focused on the low temperature regime, ${ }^{7,8}$ its possible use as a material basis for future electronic devices calls for a deeper understanding of the room temperature behavior. $^{9-13}$
In this work, we calculate the effects of remote phonon scattering on the dynamics of electrons in graphene. ${ }^{14}$ Unlike other scattering mechanisms such as disorder ${ }^{15-19}$ or acoustic phonon scattering, ${ }^{20}$ the microscopic parameters of the remote electron-phonon interaction are known a priori from independent measurements, so that, in principle, its effects can be quantitatively estimated. We show that in epitaxial graphene grown on $\mathrm{SiC}$, the polar phonon scattering has only a weak effect on the electron mobility due to the weak polarizability of the substrate and the relatively high phonon frequencies associated with the hard $\mathrm{Si}-\mathrm{C}$ bonds. The effect is much larger at graphene and/or $\mathrm{SiO}_{2}$ interfaces where, at room temperature and at the typical densities $n \geqslant 10^{12} \mathrm{~cm}^{-2}$ attained in current experiments, it gives rise to resistivities of the order of $100 \Omega$. Comparable results are expected if the substrate is polymethyl methacrylate (PMMA), ${ }^{12}$ although in that case the resistivity has a weaker temperature dependence, which is associated with the continuum of lowfrequency excitations that determine the dielectric polarizability of such amorphous material. ${ }^{21}$ These results suggests that, as in traditional $\mathrm{Si}$ based electronic devices, remote scattering with the substrate phonons can indeed constitute an important limiting factor of the mobility in future graphene devices.

\section{MODEL}

\section{A. Dielectric properties of the interface}

Insulating substrates induce fluctuating electric fields that can couple to, and partially screen, external perturbations. In bulk dielectrics, such screening properties are characterized by the frequency dependence of the dielectric constant, which can be written as

$$
\epsilon(\omega)=\epsilon_{\infty}+\sum_{i} f_{i} \frac{\omega_{T O}^{(i)^{2}}}{\omega_{T O}^{(i)^{2}}-\omega^{2}} .
$$

Here, $\epsilon_{\infty}$ is the dielectric constant at high frequencies, $\omega_{T O}^{(i)}$ denote the transverse optical modes of the dielectric, and the dimensionless quantities $f_{i}$ measure the contribution of each 
mode to the screening properties of the material. The static dielectric constant is $\epsilon_{s} \equiv \epsilon(0)$.

When a graphene sheet is deposited on a dielectric substrate, the electron charges couple to the polar modes that arise at the surface of the substrate ${ }^{1-3,22}$ (the bulk polarization vanishes outside the dielectric). Neglecting the dielectric response of the atomically thin graphene layer, the frequencies $\omega_{s}^{(i)}$ of the corresponding surface modes are determined by the equation

$$
\epsilon(\omega)+1=0,
$$

where 1 represents the dielectric constant of air. When only one polar mode is present, the above equation leads to the simple result,

$$
\omega_{s}=\omega_{T O}\left(\frac{1+\epsilon_{s}}{1+\epsilon_{\infty}}\right)^{1 / 2} .
$$

In this case, the frequency of the surface mode lies between the transverse frequency $\omega_{T O}$ and the longitudinal frequency $\omega_{L O}=\omega_{T O}\left(\epsilon_{S} / \epsilon_{\infty}\right)^{1 / 2}$ of the bulk. When more than one polar mode contribute to the dielectric constant, the above equation [Eq. (2)] must be solved by taking into account the specific values of the oscillator strengths $f_{i}$. The latter can be directly inferred from the experimental data by appropriately defining the "intermediate dielectric constants" $\epsilon_{i}$, which are evaluated at frequencies just above each resonance $\omega_{T O}^{(i)}$, as described in Ref. 3. The oscillator strengths are then given by the differences $f_{i}=\epsilon_{i-1}-\epsilon_{i}$, whose sum equals $\epsilon_{s}-\epsilon_{\infty}$.

\section{B. Remote phonon scattering}

The coupling between the electrons in graphene and the field induced by a surface mode of the dielectric is described by the following Hamiltonian:

$$
\mathcal{H}_{e-p h}=\sum_{\vec{q}} M_{\vec{q}} \rho_{\vec{q}}\left(b_{\vec{q}}^{\dagger}+b_{-\vec{q}}\right),
$$

where $b_{\vec{q}}^{\dagger}$ and $b_{-\vec{q}}$ are creation and destruction operators for surface phonons with momentum $\vec{q}$ parallel to the graphenesubstrate interface, and the electron density operator $\rho_{\vec{q}}$ is the Fourier transform of

$$
\rho(\vec{r})=\sum_{i=K, K^{\prime}} \Psi_{A_{i}}^{\dagger}(\vec{r}) \Psi_{A_{i}}(\vec{r})+\Psi_{B_{i}}^{\dagger}(\vec{r}) \Psi_{B_{i}}(\vec{r}) .
$$

As customary, here, the indices $K, K^{\prime}$ denote the two nonequivalent valleys and $A, B$ denote the two graphene sublattices. The interaction matrix element $M_{\vec{q}}$ is defined as ${ }^{1-3,22}$

$$
M_{\vec{q}}=\sqrt{g} \frac{e^{-q z}}{\sqrt{q}},
$$

where $g$ is a dimensionless coupling parameter defined below. From Eq. (6), we see that the potential induced by a polar mode of momentum $\vec{q}$ decays as $e^{-2 q z} / q$. Due to the finite distance between the substrate and the graphene layer, this potential is smooth over lengths comparable to the lattice constant of graphene, which allows us to neglect intervalley scattering.
The dimensionless coupling parameter $g$ introduced in Eq. (6) is given by

$$
g=2 \pi \beta \frac{\hbar \omega_{s}}{\hbar v_{F} / a} \frac{e^{2}}{\hbar v_{F}} .
$$

Here, $v_{F}=10^{6} \mathrm{~cm} / \mathrm{s}$ is the Fermi velocity in graphene, $a$ $=1.42 \AA$ is the $\mathrm{C}-\mathrm{C}$ bond distance, $\omega_{s}$ is the frequency of the surface phonon mode under consideration, and $z$ is the distance between the graphene layer and the substrate. The total strength of the remote phonon scattering turns out to be proportional to the parameter

$$
\beta=\frac{\epsilon_{s}-\epsilon_{\infty}}{\left(\epsilon_{s}+1\right)\left(\epsilon_{\infty}+1\right)},
$$

which is a combination of the known dielectric constants of the substrate and is a measure of the polarizability of the dielectric interface. In the following, we separately consider the case of epitaxial graphene grown on $\mathrm{SiC}$, which of graphene flakes deposited on $\mathrm{SiO}_{2}$ and on PMMA substrates.

In $6 \mathrm{H}-\mathrm{SiC}$, there is a single surface phonon mode at $\omega_{s}=116 \mathrm{meV} .{ }^{23}$ The dielectric constants of the bulk material are $\epsilon_{s}=9.7$ and $\epsilon_{\infty}=6.5$, which yields $\beta=0.040$ and $g$ $=1.7 \times 10^{-2}$.

In the case where several surface modes are present, as in $\mathrm{SiO}_{2}$, each mode couples through a partial factor $\beta_{i}$ weighted by the corresponding oscillator strength $f_{i}$ (the sum of all $\beta_{i}$ 's still given by $\beta$ defined above). For example, in crystalline $\mathrm{SiO}_{2}\left(\epsilon_{s}=3.9, \epsilon_{\infty}=2.4\right)$, the solution of Eq. (2) with the parameters given in Ref. 3 yields two dominant surface modes at $\omega_{s}^{(1)}=59 \mathrm{meV}$ and $\omega_{s}^{(2)}=155 \mathrm{meV}$, with $\beta_{1}=0.025$ and $\beta_{2}=0.062$, respectively $(\beta=0.087)$, which correspond to $g_{1}$ $=5.4 \times 10^{-3}$ and $g_{2}=3.5 \times 10^{-2}$. These values are enhanced by roughly $50 \%$ in common $\mathrm{SiO}_{2}$ glass, where $\epsilon_{\infty}=2.1$. For completeness, we also give the values for the high- $\kappa$ dielectric $\mathrm{HfO}_{2}$ that has been used in recent experiments on locally gated graphene: ${ }^{24} \epsilon_{s}=22, \epsilon_{\infty}=5, \beta=0.12$, and $\omega_{s} \simeq 94 \mathrm{meV}$, so that $g=4.2 \times 10^{-2}$.

The dielectric function of amorphous PMMA also shows a significant frequency dependence, ${ }^{21}$ varying from $\epsilon_{s}$ $\approx 2.8-3.9$ to $\epsilon_{\infty} \approx 2.2$, which signals a degree of polarizability comparable to $\mathrm{SiO}_{2}$. The modes responsible for this frequency dependence range from a continuum of anharmonic excitations at low frequencies, $0 \leq \omega \leq \omega_{c} \sim 0.02 \mathrm{eV}$, such as two level systems typical of amorphous systems, to short wavelength intramolecular phonons at higher frequencies, $\omega \gtrsim 0.15 \mathrm{eV}$. The specific features arising due to the disordered nature of PMMA will be discussed in Sec. IV D.

\section{SCATTERING RATE}

The quasiparticle scattering rate arising from the remote electron-phonon interaction described above can be calculated as

$$
\begin{aligned}
\Gamma(\omega)= & \frac{\pi}{2} \sum_{\mathbf{q}} A_{\mathbf{p}, \mathbf{q}} M_{q}^{2}\left\{\delta\left(\epsilon_{\mathbf{p}+\mathbf{q}}-\left|\omega+\omega_{s}\right|\right)\left[n_{B}+n_{F}^{+}\right]\right. \\
& \left.+\delta\left(\epsilon_{\mathbf{p}+\mathbf{q}}-\left|\omega-\omega_{s}\right|\right)\left[n_{B}+1-n_{F}^{-}\right]\right\},
\end{aligned}
$$

where the band dispersion is $\omega= \pm \epsilon_{\mathrm{p}}= \pm v_{F} p, n_{B}$ is the Bose 


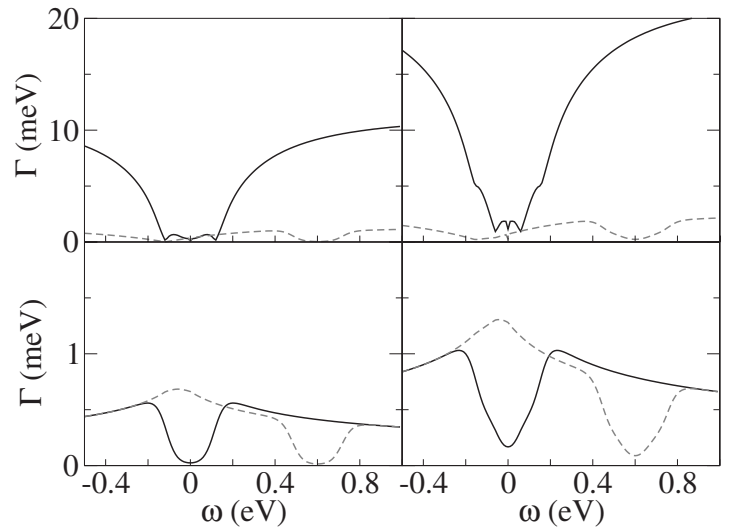

FIG. 1. Quasiparticle's scattering rate due to remote phonon interaction as a function of energy for single-layer (top) and bilayer (bottom) graphenes, with the parameters appropriate to an $\mathrm{SiC}$ (left) and a $\mathrm{SiO}_{2}$ (right) substrate, respectively. All curves are calculated at $T=300 \mathrm{~K}$. The full and dashed lines correspond, respectively, to undoped, $E_{F}=0$, and heavily doped, $E_{F}=0.6 \mathrm{eV}$ cases. Notice the different axis scales in single-layer (top) and bilayer (bottom) graphenes.

distribution for phonons of energy $\omega_{s}$, and $n_{F}^{+}, n_{F}^{-}$are the Fermi functions for electrons at $\omega \pm \omega_{s}$. The factor

$$
A_{\mathbf{p}, \mathbf{q}}=\frac{1+s \cos \left(\phi_{\mathbf{p}+\mathbf{q}}-\phi_{\mathbf{p}}\right)}{2}
$$

is the spinor overlap for intraband $(s=1)$ and interband $(s$ $=-1$ ) scatterings, respectively, where $\phi_{\mathbf{p}}$ defines the direction of $\mathbf{p}$. To a first approximation, dynamic screening from the conduction electrons can be accounted for by replacing $1 / q$ $\rightarrow 1 /\left(q+q_{s}\right)$ in the interaction matrix element $M_{q}^{2}$, where $q_{s}$ $=4 e^{2} k_{F} / \hbar v_{F}$ is the Thomas-Fermi screening length. ${ }^{25}$ This approximation assumes that the electrons instantaneously respond to the lattice vibrations and therefore constitutes a lower bound to the scattering rate. An upper bound is obtained by plainly neglecting screening. In the regime of interest here, this leads to resistivities approximately four times larger than the ones shown.

We set the graphene and/or substrate distance to the representative value $z=4 \AA$. At the typical thicknesses of the substrates used in current experiments (several hundreds of nanometers), the screening by the metallic gate can be fully neglected. Also note that our calculations do not include other effects that could modify the dielectric properties of the interface, such as the presence of a layer of $\mathrm{H}_{2} \mathrm{O} .{ }^{26}$

Equation (9) can be directly generalized to bilayer graphene by considering a parabolic band dispersion $\epsilon_{p}$ $=v_{F}^{2} p^{2} / t_{\perp}$, with $t_{\perp} \sim 0.35 \mathrm{eV}$ as the interlayer hopping parameter. In that case, the screening wave vector becomes independent of band filling, $q_{s}=4 t_{\perp} / \hbar v_{F}$, and the spinor overlap changes to $\left[1+s \cos 2\left(\phi_{\mathbf{p}+\mathbf{q}}-\phi_{\mathbf{p}}\right)\right] / 2$. We also take a slightly larger separation $z \simeq 6 \AA$, which corresponds to the average distance of the bilayer to the interface, although, in principle, the electron-phonon coupling differentiates between the two graphene sheets.

In Fig. 1, we show plots of the scattering rate $\Gamma$ corresponding to single-layer and bilayer graphenes on the two different substrates $\mathrm{SiC}$ and $\mathrm{SiO}_{2}$ for both undoped $\left(E_{F}=0\right)$ and heavily doped $\left(E_{F}=0.6 \mathrm{eV}\right)$ graphene. In all of the cases, the scattering rate is suppressed in a window $\pm \omega_{s}$ around the Fermi energy (where it actually vanishes at $T=0$ because in that case, phonon exchange is forbidden in all this range). The absolute value of $\Gamma$ at the chemical potential, which enters in the calculation of the dc conductivity, is proportional to both the coupling strength $g$ and the phonon thermal population, i.e., $\Gamma \propto g e^{-\omega_{s} / T}$. The anomalously large scattering rates obtained for undoped single-layer graphene (full lines in the upper panels of Fig. 1) compared to all of the other cases are related to the poor screening properties of Dirac electrons close to the neutrality point. Note that, although the scattering rates calculated here are of comparable magnitude as those arising from the intrinsic in-plane vibrations of graphene,${ }^{27}$ remote phonons have a much stronger influence on electronic transport due to the smaller oscillation frequencies involved (see below).

\section{CONDUCTIVITY}

\section{A. Boltzmann equation}

We calculate the electrical conductivity through the Boltzmann equation. Taking into account spin and valley degeneracy, the dc conductivity of single-layer graphene can be expressed as

$$
\sigma=\frac{e^{2}}{h} \int d \omega|\omega| \Gamma_{t r}^{-1}(\omega)\left(-\frac{d n_{F}}{d \omega}\right) .
$$

Here, $\Gamma_{t r}(\omega)$ is the transport scattering rate, which is defined as in Eq. (9), with the additional angular factor [1 $\left.-s \cos \left(\phi_{\mathbf{p}+\mathbf{q}}-\phi_{\mathbf{p}}\right)\right]$ in the integrand that favors large angle scattering events. ${ }^{28}$ Note that this approximation is strictly valid only in the elastic limit $\omega_{s} \rightarrow 0$ (where $\Gamma_{t r} \approx \Gamma / 2$ ), while it slightly underestimates the scattering rate in the inelastic regime $\omega \lessgtr \omega_{s}$. In the case of bilayer graphene, the integral in Eq. (11) has an additional factor $2 \omega / t_{\perp}$ arising from the parabolic band dispersion.

We shall present results for the conductivity of graphene on $\mathrm{SiO}_{2}$ substrates, where most experimental transport measurements have been performed (the predicted effect at room temperature is 1 order of magnitude smaller in the case of $\mathrm{SiC})$. From Eq. (11), since the derivative of the Fermi function selects contributions close to the Fermi energy, we see that the conductivity is inversely proportional to the scattering rate at $\omega \simeq E_{F}$, which, in turn, scales as $\Gamma \propto g n_{B}(T)$ $\propto \omega_{s} /\left(e^{\omega_{s} / T}-1\right)$ [see Eq. (7) and the discussion at the end of Sec. III]. At a given temperature $T$, this is a decreasing function of $\omega_{s}$ and exponentially vanishes for phonon frequencies $\omega_{s} \gg T$. As a result, the phonons that mostly affect transport are the ones with the lowest frequencies. In particular, in the case of a $\mathrm{SiO}_{2}$ substrate, the dominant phonon is the one at $\omega_{s}^{(1)}=59 \mathrm{meV}$, even though it has a weaker coupling strength. Due to the exponential temperature dependence of the Bose factor, scattering from the phonon at $\omega_{s}^{(2)}$ $=155 \mathrm{meV}$ is mostly inactive at room temperature. For the same reason, in-plane optical phonons whose characteristic energy scale is $\sim 200 \mathrm{meV}$ should give a negligible contribution to the resistivity at room temperature. 


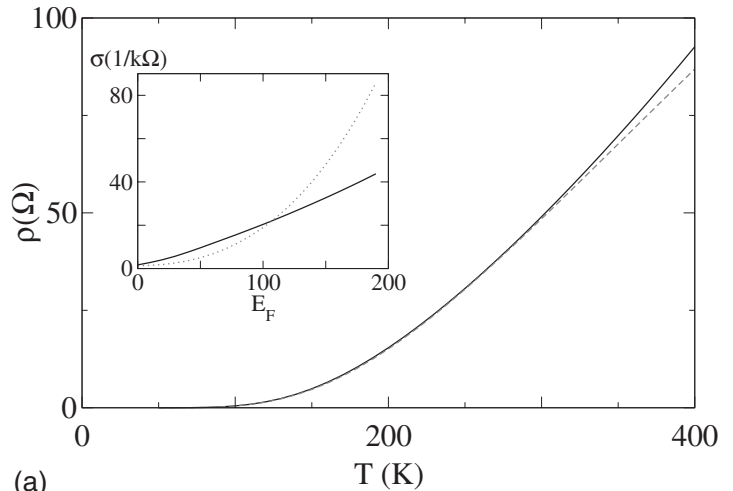

(a)

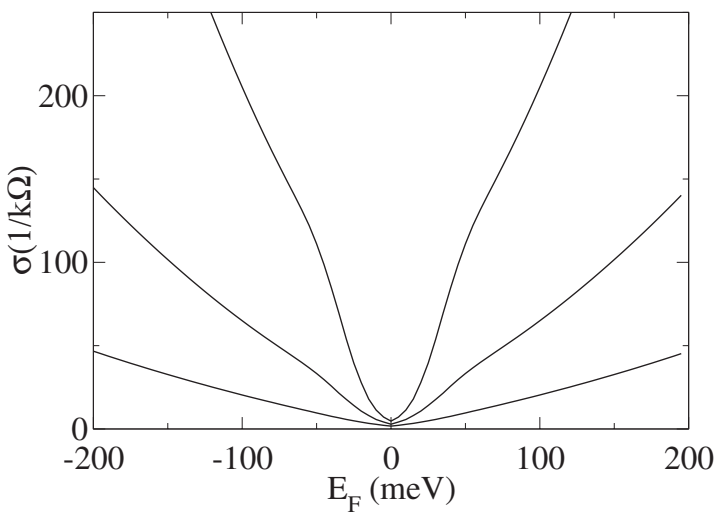

(b)

FIG. 2. (a) Temperature dependence of the resistivity for $E_{F}$ $=100 \mathrm{meV}$ (the dashed line corresponds to $\rho=\rho_{0} e^{-\omega_{s 1} / T}$, with $\rho_{0}$ $=500 \Omega$ ). (b) Conductivity vs chemical potential for $T=150,200$, and $300 \mathrm{~K}$ (from top to bottom). The inset of (a) shows the comparison with bilayer graphene (dotted line) at $T=300 \mathrm{~K}$.

\section{B. Substrate-limited conductivity}

In Fig. 2(a), we show the result of $\rho$ vs $T$ at $E_{F}$ $=100 \mathrm{meV}$ (corresponding to a density $n=0.7 \times 10^{12} \mathrm{~cm}^{-2}$ ), obtained by setting a finite distance $z=4 \AA$ between the substrate and the graphene layer and a Thomas-Fermi screening from the graphene electrons as described above. As anticipated from the preceding discussion, the resistivity exhibits a characteristic exponential behavior, which arises from the phonon thermal population. Note that unlike the short-range scattering from in-plane phonons, the present result has a sizable density dependence due to the long-range nature of the interaction. This is illustrated in Fig. 2(b), where we plot the dependence of $\sigma$ on $E_{F}$ at fixed $T=300,200$, and $150 \mathrm{~K}$ (from bottom to top). In the explored density range, the conductivity has an overall linear dependence on $E_{F}$ away from the Dirac point, which is intermediate between the cases of short-range scatterers $(\sigma \sim$ const $)$ and charged impurities $(\sigma$ $\left.\propto E_{F}^{2}\right) .{ }^{15}$ This behavior can be understood from standard dimensional arguments, observing that the scattering rate of Eq. (9) scales as $M_{q \simeq k_{F}}^{2} \propto k_{F}^{-1}$ times the density of states $\propto k_{F}$, which yields $\Gamma \sim$ const. The result $\sigma \propto\left|E_{F}\right|$ then directly follows from Eq. (11). Note that the zero temperature "universal" value $\sigma_{\min }=4 e^{2} / h \pi$ would not be recovered upon approaching the Dirac point, even using a more accurate treatment beyond the Boltzmann equation. The reason is that the rate $\Gamma$ arising from the scattering to optical phonons is

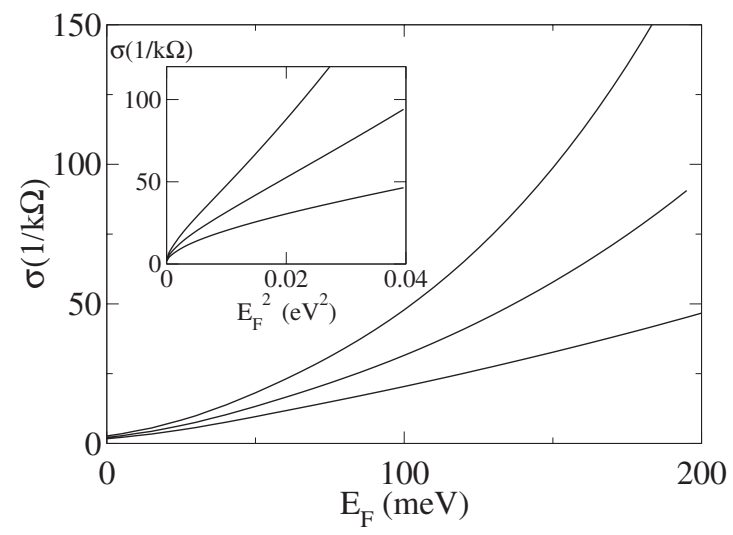

FIG. 3. Same as Fig. 2(b) for different values of the distance $z$. From bottom to top, $z=4,12$, and $20 \AA$. A crossover to a superlinear behavior is apparent at large $z$ and large $E_{F}$. In the inset, the same data are plotted as a function of $E_{F}^{2} \propto n$.

always much (exponentially) smaller than the temperature itself, while the universal result is obtained in the opposite limit $T \ll \Gamma{ }^{17}$

Superimposed to the smooth density dependence analyzed above, a slight "kink" appears in the electrical characteristic at $E_{F} \simeq \omega_{s}^{(1)}$ that testifies the interaction with the sharp interface phonon mode. The anomaly becomes more pronounced at low temperatures where, however, the absolute value of the scattering rate becomes extremely small, and it is likely to be completely hidden by the presence of other sources of scattering. Note also that the Boltzmann treatment used here is probably inappropriate in the region close to the Dirac point.

In the inset of Fig. 2(b), we compare the conductivity for single-layer and bilayer graphenes at $T=300 \mathrm{~K}$. The different densities of states in bilayer graphene leads to a stronger energy dependence. Accordingly, bilayer graphene should be a better conductor at large bias. With the present choice of parameters, the crossing occurs at $E_{F} \sim 0.1 \mathrm{eV}$.

\section{Density dependence}

The simple scaling argument presented above, which leads to a linear dependence $\sigma \propto\left|E_{F}\right|$ for single-layer graphene, only holds when the distance $z$ is sufficiently small and breaks down when $2 k_{F} z \sim 1$. At larger distances, the integrals over momenta required to calculate the scattering rate are limited by a cutoff $q_{\max } \sim z^{-1}$. As a result, scattering is only allowed between electrons with similar momenta. For these processes, the suppression of backscattering typical of graphene, associated with the overlap factor $\left[1 \pm \cos \left(\phi_{\mathbf{p}+\mathbf{q}}\right.\right.$ $\left.-\phi_{\mathbf{p}}\right)$ ], does not play a role. As consequence, the scattering rate grows faster with $k_{F}$ than in the limit $z \rightarrow 0$, where $q_{c}$ can be neglected. The precise dependence on $k_{F}$ is determined by the singular behavior of the scattering matrix element at low momenta, $\left|M_{q}\right|^{2} \propto q^{-1}$. Eventually, we find that at large $z$, the scattering rate $\Gamma \propto\left|E_{F}\right|^{3}$, which leads to a conductivity $\sigma \propto E_{F}^{4}$.

In Fig. 3, we show plots of $\sigma$ vs $E_{F}$ at $T=300 \mathrm{~K}$ for increasing values of $z$. While for $z=4 \AA$ the behavior is ap- 
proximately linear in the whole energy range, a clear upturn becomes visible at larger separations, which reflects the expected crossover at $\left|E_{F}\right| z / v_{F} \sim 1 / 2$. Notably, at intermediate values of the distance $z$, this phenomenon can lead to an apparently linear density dependence, i.e., $\sigma \propto E_{F}^{2} \propto n,{ }^{13}$ which is best seen in the inset of Fig. 3 .

\section{PMMA substrates}

The broad continuum of modes that contributes to the dielectric polarizability of PMMA does not allow us to provide accurate analytical expressions for the scattering rate in that case. Nevertheless, an order-of-magnitude estimate of the effects arising from this substrate at room temperature can still be obtained. It was noted in Sec. III that each phonon mode gives rise to a scattering rate proportional to the product of the coupling constant $g \propto \beta \omega_{s}$ times the thermal occupation factor $n_{B}$, i.e., $\Gamma \propto \beta \omega_{s} n_{B}$. Summing over all the modes yields

$$
\Gamma \approx \sum_{i} n_{B}^{i} \beta_{i} \omega_{s}^{(i)}
$$

At temperatures above the range of the low-frequency modes, determined by the cutoff frequency $\omega_{c} \sim 0.02 \mathrm{eV}$, the corresponding Bose factors become $n_{B} \simeq T / \omega_{s}^{i}$. Neglecting contributions from the high-frequency vibrations, the phonon frequencies thus cancel out from the sum in Eq. (12), which leads to

$$
\Gamma \approx T \sum_{i}^{\omega_{s}^{(i)}<\omega_{c}} \frac{\epsilon_{i-1}-\epsilon_{i}}{\left(\epsilon_{s}+1\right)\left(\epsilon_{\infty}+1\right)}=T \frac{\epsilon_{s}-\epsilon\left(\omega_{c}\right)}{\left(\epsilon_{s}+1\right)\left(\epsilon_{\infty}+1\right)} .
$$

In this high temperature regime, the scattering rate arising from the PMMA substrate only depends on the dielectric constants of the material, being proportional to the difference $\left[\epsilon_{0}-\epsilon\left(\omega_{c}\right)\right] \approx 0.1-0.2$.

Up to numerical factors, the corresponding resistivity is approximately given by $\rho \approx\left(h / e^{2}\right)\left(\Gamma / E_{F}\right)$ [see Eq. (11)], which leads to

$$
\rho \approx\left(\frac{h}{e^{2}}\right) \frac{\epsilon_{s}-\epsilon\left(\omega_{c}\right)}{\left(\epsilon_{s}+1\right)\left(\epsilon_{\infty}+1\right)} \frac{T}{E_{F}} .
$$

The above result shows that, since the phonon frequencies involved in the scattering process are small compared to the temperature, the exponential temperature dependence characteristic of $\mathrm{SiO}_{2}$ substrates converts into a smoother powerlaw dependence. Nevertheless, even though the remote electron-phonon coupling strength in the case of PMMA is much less than that of a $\mathrm{SiO}_{2}$ substrate (in $\mathrm{SiO}_{2} \epsilon_{s}-\epsilon_{\infty}$ $\simeq 1.5$ ), the phonon induced resistivity turns out to have a comparable magnitude, precisely due to the smaller oscilla- tion frequencies. Indeed, for $T \approx 300 \mathrm{~K}$ and $E_{F} \approx 0.1 \mathrm{eV}$, Eq. (14) gives $\rho \sim 50-100 \Omega$.

\section{DISCUSSION AND CONCLUSIONS}

We have estimated the quasiparticle scattering rates induced by the polar modes of polarizable substrates such as $\mathrm{SiO}_{2}, \mathrm{SiC}$, and PMMA on single-layer and bilayer graphenes. Our results show that, while remote phonon scattering has negligible effects on transport at low temperatures, it becomes relevant at room temperature, where it could constitute the dominant limiting factor of the electron mobility in sufficiently clean samples, especially for $\mathrm{SiO}_{2}$ and PMMA substrates. Larger effects can be expected when substrates with a higher degree of polarizability are used as gate dielectrics. The effects of remote phonon scattering are slightly reduced in doped bilayer graphene, due to the more conventional parabolic band dispersion, which makes it more suitable for device applications at room temperature.

Although the transport properties of graphene are essentially temperature independent below $T=100 \mathrm{~K}$, recent experiments performed in field-effect doped samples deposited on dielectric substrates have reported a sharp increase in the resistivity of the order of $\Delta \rho \sim 100 \Omega$ around room temperature at typical densities $n \gtrsim 10^{12} \mathrm{~cm}^{-2} \cdot{ }^{9-13}$ This increase is unlikely to be due to the effects of disorder, which are expected to smoothly depend, if at all, on the temperature. Also the in-plane optical phonons of graphene can be ruled out due to their high oscillation frequencies, as discussed in Sec. III. That short-range scattering from in-plane deformations of the lattice is not the main cause of the observed behavior is further supported by the observation that the temperature dependent contribution to the resistivity is not independent of density, ${ }^{12,13}$ pointing to a long-range scattering mechanism such as the one studied here. Our results indicate that the recently measured density and temperature dependent contribution to the resistivity are compatible with the mechanism of remote phonon scattering.

According to the present scenario, the maximum conductivity that can be achieved in a given sample crucially depends on the dielectric properties of the gate insulator. Therefore, the use of nonpolar substrates or suspended samples ${ }^{29,30}$ should be favored to optimize the transport properties of graphene devices.

\section{ACKNOWLEDGMENTS}

This work was supported by MEC (Spain) through Grant No. FIS2005-05478-C02-01, the Comunidad de Madrid, through the program CITECNOMIK Grant No. CM2006-S0505-ESP-0337, and the European Union under Contract No. 12881 (NEST). The authors thank M.S. Fuhrer for stimulating correspondence and T. Stauber for useful discussions. 
${ }^{1}$ S. Q. Wang and G. D. Mahan, Phys. Rev. B 6, 4517 (1972).

${ }^{2}$ K. Hess and P. Vogl, Solid State Commun. 30, 807 (1979).

${ }^{3}$ M. V. Fischetti, D. A. Neumayer, and E. A. Cartier, J. Appl. Phys. 90, 4587 (2001).

${ }^{4}$ R. Chau, S. Datta, M. Doczy, B. Doyle, J. Kavalieros, and M. Metz, IEEE Electron Device Lett. 25, 408 (2004).

${ }^{5}$ A. F. Stassen, R. W. I. de Boer, N. N. Iosad, and A. F. Morpurgo, Appl. Phys. Lett. 85, 3899 (2004).

${ }^{6}$ I. N. Hulea, S. Fratini, H. Xie, C. L. Mulder, N. N. Iossad, G. Rastelli, S. Ciuchi, and A. F. Morpurgo, Nat. Mater. 5, 982 (2006).

${ }^{7}$ A. K. Geim and K. S. Novoselov, Nat. Mater. 6, 183 (2007).

${ }^{8}$ A. H. Castro Neto, F. Guinea, N. M. R. Peres, K. S. Novoselov, and A. K. Geim, arXiv:0709.1163, Rev. Mod. Phys. (to be published).

${ }^{9}$ E. W. Hill, A. K. Geim, K. S. Novoselov, F. Schedin, and P. Blake, IEEE Trans. Magn. 42, 2694 (2006).

${ }^{10}$ Y.-W. Tan, Y. Zhang, H. L. Störmer, and P. Kim, Eur. Phys. J. Spec. Top. 148, 15 (2007).

${ }^{11}$ Y.-W. Tan, Y. Zhang, K. Bolotin, Y. Zhao, S. Adam, E. H. Hwang, S. Das Sarma, H. L. Störmer, and P. Kim, Phys. Rev. Lett. 99, 246803 (2007).

${ }^{12}$ S. V. Morozov, K. S. Novoselov, M. I. Katsnelson, F. Schedin, D. Elias, J. A. Jaszczak, and A. K. Geim, Phys. Rev. Lett. 100, 016602 (2008).

${ }^{13}$ J. H. Chen, C. Jang, S. Xiao, M. Ishigami, and M. S. Fuhrer, Nat. Nanotechnol. 3, 206 (2008).

${ }^{14}$ K. S. Novoselov, D. Jiang, F. Schedin, T. J. Booth, V. V. Khotkevich, S. V. Morozov, and A. K. Geim, Proc. Natl. Acad. Sci. U.S.A. 102, 10451 (2005).
${ }^{15}$ K. Nomura and A. H. MacDonald, Phys. Rev. Lett. 98, 076602 (2007).

${ }^{16}$ S. Adam, E. H. Hwang, V. M. Galitski, and S. Das Sarma, Proc. Natl. Acad. Sci. U.S.A. 104, 18392 (2007).

${ }^{17}$ K. Ziegler, Phys. Rev. B 75, 233407 (2007).

${ }^{18}$ T. Stauber, N. M. R. Peres, and F. Guinea, Phys. Rev. B 76, 205423 (2007).

${ }^{19}$ M. I. Katsnelson and A. K. Geim, Philos. Trans. R. Soc. London, Ser. A 366, 195 (2008).

${ }^{20}$ E. H. Hwang and S. Das Sarma, Phys. Rev. B 77, 115449 (2008).

${ }^{21}$ S. Kojima, M. Wada Takeda, and S. Nishizawa, J. Mol. Struct. 651, 285 (2003).

${ }^{22}$ N. Mori and T. Ando, Phys. Rev. B 40, 6175 (1989).

${ }^{23}$ H. Nienhaus, T. U. Kampen, and W. Mönch, Surf. Sci. Lett. 324, L328 (1989).

${ }^{24}$ B. Özyilmaz, P. Jarillo-Herrero, D. Efetov, and P. Kim, Appl. Phys. Lett. 91, 192107 (2007).

${ }^{25}$ B. Wunsch, T. Stauber, F. Sols, and F. Guinea, New J. Phys. 8, 318 (2006).

${ }^{26}$ J. Sabio, C. Seoánez, S. Fratini, F. Guinea, A. H. Castro Neto, and F. Sols, Phys. Rev. B 77, 195409 (2008).

${ }^{27}$ F. Guinea, J. Phys. C 14, 3345 (1981).

${ }^{28}$ G. D. Mahan, Many Particle Physics, 2nd ed. (Plenum, New York, 1990).

${ }^{29}$ K. I. Bolotin, K. J. Sikes, Z. Jiang, G. Fudenberg, J. Hone, P. Kim, and H. L. Stormer, arXiv:0802.2389 (unpublished).

${ }^{30}$ X. Du, I. Skachko, A. Barker, and E. Y. Andrei, arXiv:0802.2933 (unpublished). 